\title{
Simple Pneumonia or Something More?: A Case Report and Discussion of Unexpected Empyema Identified by Point-of-Care Ultrasound
}

\author{
Michael Romano, MD*; Tomislav Jelic, $\mathrm{MD}^{\dagger}$; Jordan Chenkin, MD, MEd ${ }^{*}$
}

\section{ABSTRACT}

There is evidence to suggest that point-of-care ultrasound assessment of the lungs has a higher sensitivity and specificity than chest radiography for the diagnosis of pneumonia. It is unknown if the same is true for pneumonia complications. We present and discuss the case of a 61-yearold woman who presented to the emergency department with confusion, decreased level of consciousness, and signs of sepsis. A chest $x$-ray revealed a right sided infiltrate. An ultrasound of the patient's lungs was performed, and revealed a complex loculated fluid collection consistent with an empyema. A chest CT confirmed the diagnosis, and immediate percutaneous drainage was performed.

\section{RÉSUMÉ}

Certaines données autorisent à penser que l'échographie pratiquée au point de service aurait une sensibilité et une spécificité plus grandes que la radiographie des poumons dans le diagnostic de la pneumonie. Toutefois, l'on ne sait pas si la même observation vaut pour les complications de la pneumonie. Sera exposé ici le cas d'une femme de 61 ans, examinée au service des urgences pour de la confusion, une diminution de l'état de conscience et des signes de sepsie; suivra une discussion. Une radiographie des poumons a révélé la présence d'infiltration dans les tissus du côté droit. De son côté, l'échographie a montré une image d'une collection liquidienne loculée complexe, compatible avec celle d'un empyème. Une tomodensitométrie des poumons a permis de confirmer le diagnostic, et on a aussitôt procédé au drainage percutané.

Keywords: PoCUS, lung ultrasound, emphyema

\section{INTRODUCTION}

A growing body of evidence suggests that lung ultrasound can be a useful procedure for assessing patients with respiratory complaints. The most well-known study of this is the "BLUE Protocol," reported by
Lichtenstein in 2008. This study found that lung ultrasound in the intensive care unit was highly accurate in differentiating causes of respiratory failure, including congestive heart failure, pneumothorax, acute respiratory distress syndrome, and pneumonia. ${ }^{1}$ Subsequent to this report, numerous studies have shown that emergency physicians can accurately apply lung ultrasound in the emergency department (ED). Zanobetti et al. found that in $404 \mathrm{ED}$ patients presenting with acute dyspnea, point-of-care ultrasound (PoCUS) was on average 95 minutes faster than a chest $x$-ray, and had a higher agreement rate with chest CT findings than did chest $\mathrm{x}$-ray. ${ }^{2}$ PoCUS for pneumonia is also well studied. In a recent systematic review comparing ultrasound with chest CT, lung ultrasound in adults was found to have a sensitivity of $94 \%$ and a specificity of $96 \%$ for pneumonia. ${ }^{3}$ For patients arriving with undifferentiated shortness of breath and sepsis, PoCUS may be a valuable tool to help to rapidly diagnose the presence of important lung and pleural pathology.

\section{CASE REPORT}

A 61-year-old woman was transported to the ED, by paramedics, with confusion and a decreased level of consciousness. Her family indicated she had a two-week history of worsening minimally productive cough without hemoptysis, fatigue, weakness, and mild dyspnea on exertion in the prior 24 hours. Her past medical history included rheumatoid arthritis and Sjogren syndrome, for which she was on no medical therapy. She had no prior history of congestive heart failure or restrictive lung disease and no recent history of travel or unusual exposures. She was a non-smoker,

From the *University of Toronto, Toronto, ON; and TSunnybrook Health Sciences Centre, Toronto, ON.

Correspondence to: Dr. Tomislav Jelic, One Davis Boulevard, Suite 503, Tampa, FL 33606; Email: tjelic@gmail.com

(C) Canadian Association of Emergency Physicians

CJEM 2016;18(5):391-394

DOI 10.1017/cem.2015.71 


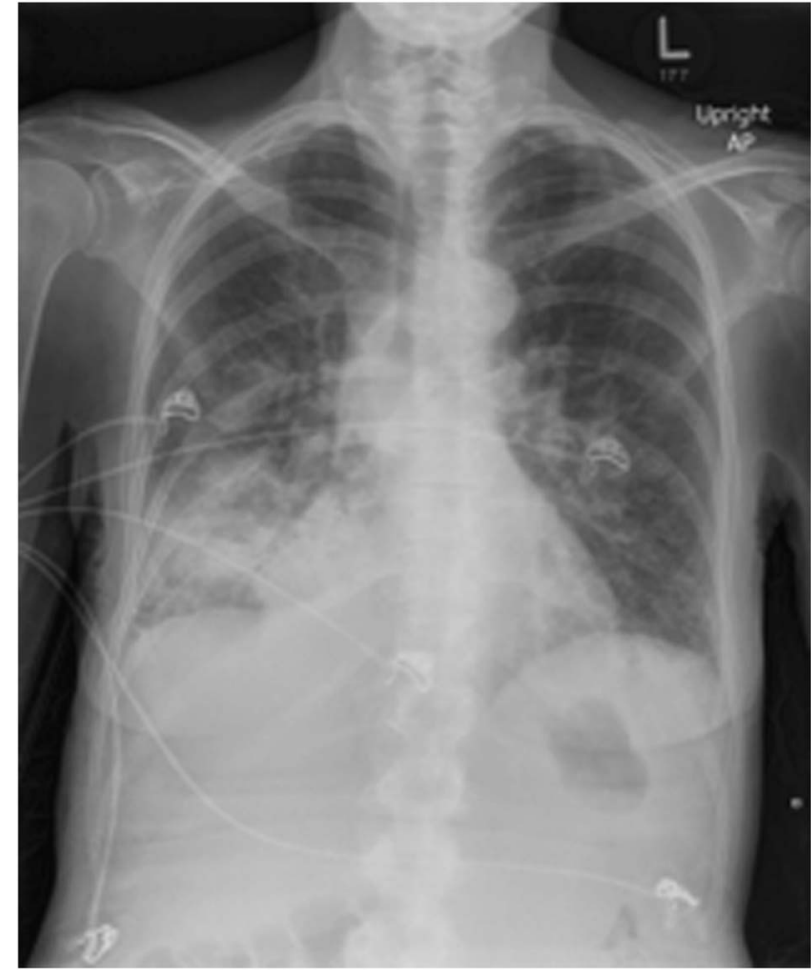

Figure 1. Chest $X$-ray, demonstrating a right infiltrate.

did not consume alcohol, and was normally highfunctioning. The decline in her functional status in the past several days had been precipitous.

Her initial triage vitals were: heart rate of 140 beats per minute, blood pressure of 91/61 $\mathrm{mm} \mathrm{Hg}$, respiratory rate 25 breaths per minute, temperature of $39^{\circ} \mathrm{C}$ $\left(102.2^{\circ} \mathrm{F}\right)$, room air oxygen saturation of $86 \%$. Physical examination revealed the patient was drowsy but easily roused. Head and neck examination was normal. Cardiac examination was unremarkable, with no murmurs, rubs, or thrills. Respiratory examination revealed decreased air entry to the right base. A portable chest $\mathrm{x}$-ray was performed (Figure 1). This demonstrated an extensive heterogenous and poorly defined rightmiddle lobe consolidation. Intravenous antibiotics were commenced and the patient was referred to internal medicine for further management for a presumed right-middle lobe pneumonia.

Due to the lung consolidation visible on chest $\mathrm{x}$-ray, and subsequent to the decision to commence IV antbiotics and admit the patient, we performed a pointof-care ultrasound (PoCUS) of the patient's thorax. In the right posterolateral region, we identified a complex loculated pleural fluid collection suggesting the presence of an empyema (Figures 2 and 3). The



Figure 2. PoCUS of the right chest, demonstrating a complex, cystic, structure. Findings in keeping with an empyema.

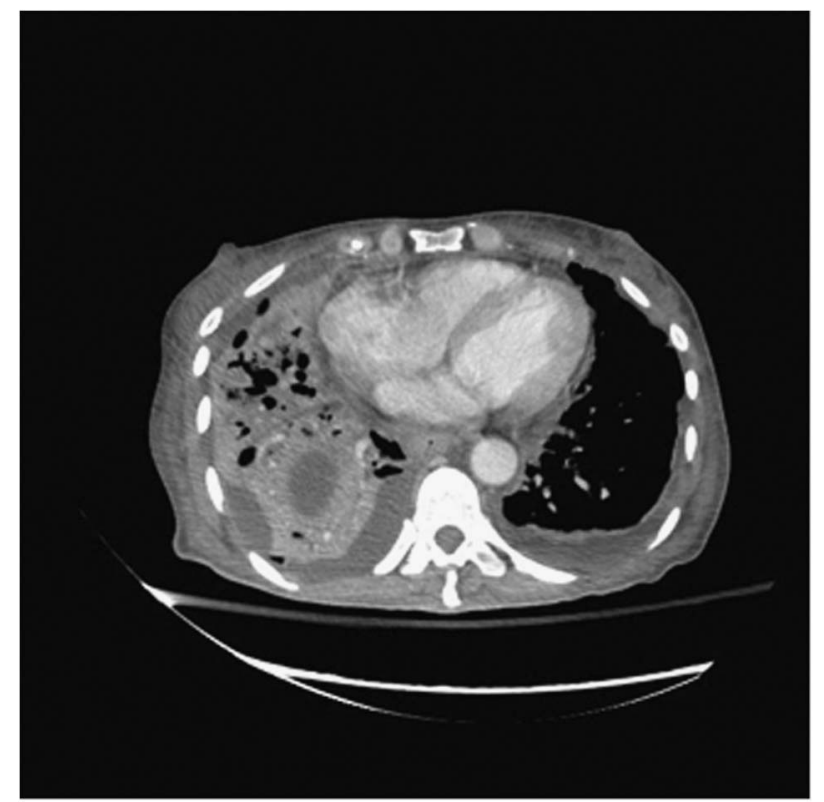

Figure 3. CT Chest, demonstrating the complex empyema seen on PoCUS.

findings were reported to internal medicine and a thoracic CT was ordered for further characterization of the collection. The CT confirmed a complex loculated fluid collection consistent with an empyema (Figure 4).

The patient underwent immediate chest tube drainage of the empyema. Her blood cultures and empyema fluid cultures were both positive for Streptococcus pneumoniae and she was admitted to hospital for approximately two weeks. She was then transferred to another institution with thoracic surgery capabilities, as her subsequent chest CTs demonstrated incomplete drainage of the empyema collection; however, no surgery was ultimately required. 


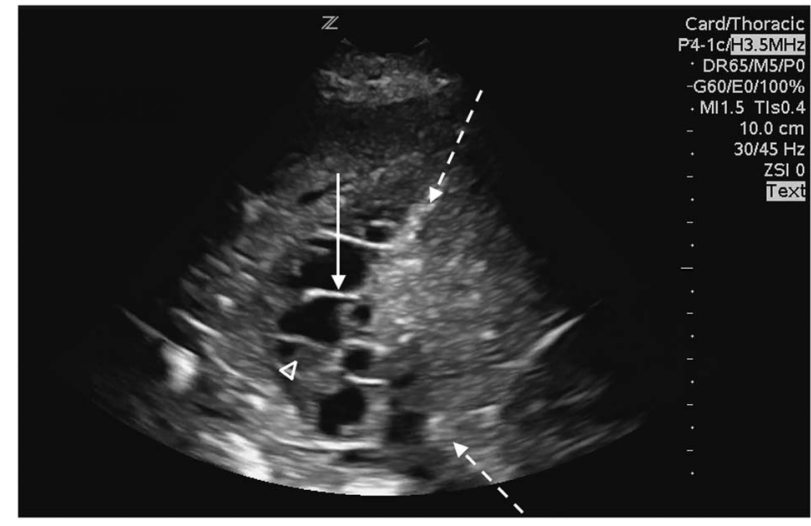

Figure 4. Dashed arrow: Representing the hyper echoic diaphragm. Solid arrow: Septation present within the cystic structure. Arrow head: Echogenic debris within the cystic structure, suggesting possible empyema.

The patient was discharged home from hospital with a complete recovery.

\section{DISCUSSION}

Although $40 \%$ of pneumonias may be complicated with parapneumonic effusion, only $5 \%-10 \%$ go on to formation of an empyema. ${ }^{4,5}$ A study of 3,675 patients found that empyemas occurred in only $1.3 \%$ of communityacquired pneumonia cases. ${ }^{6}$ A national Canadian database found non-surgical empyema rates have increased by an incidence rate ratio of 1.30 over an nine-year period ending in 2003. ${ }^{7}$ Patients who were younger and with a history of intravenous drug use were found to be at higher risk for empyema. Common organisms implicated are Streptococcus pneumoniae, Staphylococcus aureus, and Streptococcus milleri. Anaerobic bacteria are also identified in between $36 \%$ and $76 \%$ of cultures. ${ }^{8}$

Empyema carries a mortality rate of between $4 \%$ and $7 \%$. Chalmers and colleagues prospectively identified six risk factors associated with patients admitted with community-acquired pneumonia developing a complicated parapneumonic effusion or empyema. These factors were: albumin $<30 \mathrm{~g} / \mathrm{L}$, sodium $<130 \mathrm{mmol} / \mathrm{L}$, platelet count $>400 \times 10^{9}$, C-reactive protein (CRP) $>100 \mathrm{mg} / \mathrm{L}$, and a history of alcohol or intravenous drug use. Elevated CRP and hypoalbuminemia conferred the highest adjusted odds ratios, at 15.7 and 4.6, respectively. ${ }^{9}$ Beyond situations involving the aforementioned risk factors, the diagnosis of empyema should be considered in pneumonia patients with prolonged symptoms, failure of antibiotic treatment, or failure of infiltrate resolution on radiography. ${ }^{6,9,10}$
Point-of-care ultrasound is increasingly used for the assessment of lung and pleural pathology in the ED. Recent studies have found that PoCUS has a high sensitivity and specificity for the detection of pneumonia, and may in fact be more sensitive than chest radiography. 8,9 Ultrasound findings suggesting pneumonia include a consolidated lung that takes on the appearance of a solid organ (termed "hepatization"), dynamic air bronchograms (air and fluid moving in and out of the consolidated lung), and a surrounding interstitial pattern (identified by bright lines with irregular margins spreading deep to the consolidation). ${ }^{11}$

In addition to its potential utility in diagnosing pneumonia, PoCUS may be helpful in determining whether there are associated complications such as pleural effusions or empyema. In an ICU study of 42 patients, ultrasound has been found to be superior to chest radiography for the detection of pleural fluid, with a sensitivity and specificity of $100 \%$, compared to a sensitivity and specificity of $65 \%$ and $81 \%$, respectively, for chest $\mathrm{x}$-ray. ${ }^{12}$ In addition, ultrasound has been reported to be more accurate than chest $\mathrm{x}$-ray for determining the size of the pleural effusion. ${ }^{13}$ Ultrasound findings suggestive of an empyema include a complex fluid collection in the pleural space with echogenic debris or septations. ${ }^{14}$ The British Thoracic Society Guideline on management of pleural infection in adults states that ultrasound can be used for the initial assessment of the presence of pleural effusions and empyemas. ${ }^{15}$ We were unable to identify any studies evaluating the sensitivity or specificity of PoCUS for the diagnosis of empyema.

In the case we present, a thoracic ultrasound was performed as a routine educational scan by trainees in the process of learning how to identify lung and pleural pathology. Trainees had received a didactic lecture on the background of lung ultrasound, and were on a training shift to learn the technical aspects of lung ultrasound examination. The lung ultrasound assessment was performed according to commonly used scanning protocols. ${ }^{1,16}$ The fact that the PoCUS identified an important unexpected finding in our case may suggest that emergency physicians should have a lower threshold to pursue thoracic ultrasound in patients with respiratory complaints, or when there is a consolidation identified on chest $x$-ray in the setting of risk factors for empyema. In the case we present, the patient's main risk factor for formation of an empyema was the prolonged duration of her symptoms. ${ }^{15}$ 
In addition to this, her ED blood work revealed thrombocytosis (platelet count 500) and hypoalbuminemia (albumin $21 \mathrm{~g} / \mathrm{L}$ ), both of which are predictive for possible empyema, though extremely non-specific. ${ }^{5}$

\section{CONCLUSION}

We present a case where a point-of-care thoracic ultrasound done for educational purposes identified an unsuspected empyema in a patient being treated for community-acquired pneumonia. Emergency physicians should be aware of the limitations of chest radiography for the identification of pleural effusions and empyemas, and may need to have a lower threshold for consideration of thoracic ultrasound in patients with significant respiratory complaints. In particular, pneumonia patients with prolonged symptoms, patients who fail antibiotic therapy, intravenous drug users, and patients who are immunosuppressed should be considered for point-of-care ultrasound examination of the lungs. Further studies are needed to determine the test characteristics of PoCUS for the diagnosis of empyema.

Competing Interests: None to declare.

\section{REFERENCES}

1. Lichtenstein DA, Meziere GA. Relevance of lung ultrasound in the diagnosis of acute respiratory failure: the BLUE protocol. Chest 2008;134(1):117-25.

2. Zanobetti M, Poggioni C, Pini R. Can chest ultrasonography replace standard chest radiography for evaluation of acute dyspnea in the ED? Chest 2011;139(5):1140-7.
3. Chavez MA, Shams N, Ellington LE, et al. Lung ultrasound for the diagnosis of pneumonia in adults: a systematic review and meta-analysis. Respir Res 2014;15:50.

4. Desai H, Agrawal A. Pulmonary emergencies: pneumonia, acute respiratory distress syndrome, lung abscess, and empyema. Med Clin North Am 2012;96(6):1127-48.

5. Light RW, Girard WM, Jenkinson SG, et al. Parapneumonic effusions. Am $\mathcal{F}$ Med 1980;69(4):507-12.

6. Ahmed RA, Marrie TJ, Huang JQ. Thoracic empyema in patients with community-acquired pneumonia. Am $7 \mathrm{Med}$ 2006;119(10):877-83.

7. Finley C, Clifton J, Fitzgerald JM, et al. Empyema: an increasing concern in Canada. Can Respir 7 2008;15(2):85-9.

8. Brook I, Frazier EH. Aerobic and anaerobic microbiology of empyema. A retrospective review in two military hospitals. Chest 1993;103(5):1502-7.

9. Chalmers JD, Singanayagam A, Murray MP, et al. Risk factors for complicated parapneumonic effusion and empyema on presentation to hospital with communityacquired pneumonia. Thorax 2009;64(7):592-7.

10. Sahn SA. Diagnosis and management of parapneumonic effusions and empyema. Clin Infect Dis 2007;45(11):1480-6.

11. Bourcier JE, Paquet J, Seinger $M$, et al. Performance comparison of lung ultrasound and chest $\mathrm{x}$-ray for the diagnosis of pneumonia in the ED. Am 7 Emerg Med 2014; 32(2):115-8.

12. Xirouchaki N, Magkanas E, Vaporidi K, et al. Lung ultrasound in critically ill patients: comparison with bedside chest radiography. Intensive Care Med 2011;37(9):1488-93.

13. Eibenberger KL, Dock WI, Ammann ME, et al. Quantification of pleural effusions: sonography versus radiography. Radiology 1994;191(3):681-4.

14. Yang PC, Luh KT, Chang DB, et al. Value of sonography in determining the nature of the pleural effusion: analysis of 320 cases. A7R Am 7 Roentgenol 1992;159(1):29-33.

15. Davies HE, Davies RJ, Davies CW, et al. Management of pleural infection in adults: British Thoracic Society Pleural Disease Guideline 2010. Thorax 2010;65(S2):ii41-53.

16. Gargani L, Volpicelli G. How I do it: lung ultrasound. Cardiovasc Ultrasound 2014;12:25. 